\title{
Microjets in the penumbra of a sunspot
}

\author{
Ainar Drews and Luc Rouppe van der Voort \\ Institute of Theoretical Astrophysics, University of Oslo, PO Box 1029 Blindern, 0315 Oslo, Norway \\ e-mail: ainar.drews@astro.uio.no
}

Received 21 December 2016 / Accepted 18 February 2017

\begin{abstract}
Context. Penumbral microjets (PMJs) are short-lived jets found in the penumbra of sunspots, first observed in wide-band Ca II $\mathrm{H}$ line observations as localized brightenings, and are thought to be caused by magnetic reconnection. Earlier work on PMJs has focused on smaller samples of by-eye selected events and case studies.

Aims. It is our goal to present an automated study of a large sample of PMJs to place the basic statistics of PMJs on a sure footing and to study the PMJ Ca II 8542 A spectral profile in detail.

Methods. High spatial resolution and spectrally well-sampled observations in the Ca II $8542 \AA$ line obtained from the Swedish 1-m Solar Telescope (SST) were reduced by a principle component analysis and subsequently used in the automated detection of PMJs using the simple machine learning algorithm k-nearest neighbour. PMJ detections were verified with co-temporal Ca II $\mathrm{H}$ line observations.

Results. We find a total of 453 tracked PMJ events, 4253 PMJs detections tallied over all timeframes, and a detection rate of 21 events per timestep. From these, an average length, width and lifetime of $640 \mathrm{~km}, 210 \mathrm{~km}$ and $90 \mathrm{~s}$ are obtained. The average PMJ Ca II 8542 A line profile is characterized by enhanced inner wings, often in the form of one or two distinct peaks, and a brighter line core as compared to the quiet-Sun average. Average blue and red peak positions are determined at $-10.4 \mathrm{~km} \mathrm{~s}^{-1}$ and $+10.2 \mathrm{~km} \mathrm{~s}^{-1}$ offsets from the Ca II 8542 A line core. We find several clusters of PMJ hot-spots within the sunspot penumbra, in which PMJ events occur in the same general area repeatedly over time.

Conclusions. Our results indicate smaller average PMJs sizes and longer lifetimes compared to previously published values, but with statistics still in the same orders of magnitude. The investigation and analysis of the PMJ line profiles strengthens the proposed heating of PMJs to transition region temperatures. The presented statistics on PMJs form a solid basis for future investigations and numerical modelling of PMJs.
\end{abstract}

Key words. Sun: atmosphere - Sun: chromosphere - Sun: photosphere - sunspots - Sun: magnetic fields

\section{Introduction}

Penumbral microjets (PMJs) are short-lived, elongated, transients in the chromosphere of sunspot penumbrae. They were discovered (Katsukawa et al. 2007) in Ca II H time sequences from Hinode's $3 \AA$ wide imaging filter in which PMJs display a 10-20\% brightness enhancement as compared to surrounding penumbral structures. In the Hinode observations, PMJs have typical lifetimes of up to one minute, lengths between 1000 and $4000 \mathrm{~km}$, widths of about $400 \mathrm{~km}$, and apparent rise velocity faster than $100 \mathrm{~km}^{-1}$ (Katsukawa et al. 2007).

Penumbrae are known to host strong convectively driven plasma flows and magnetic fields that vary significantly at small spatial scales, both in inclination and magnitude (see e.g., Borrero \& Ichimoto 2011). In this magnetically stressed environment, magnetic reconnection appears to be a viable candidate as driver of PMJs. This is supported by the measurements of the apparent inclination of PMJs with respect to the photospheric penumbral filaments (Katsukawa et al. 2007) and magnetic fields (Jurčák \& Katsukawa 2008). Further indications of the reconnection scenario come from Katsukawa \& Jurčák (2010) who report the association of small photospheric downflow patches with some PMJs. These could be interpreted as the downward flows from magnetic reconnection above the photosphere. Evidence of progressive heating along PMJs is reported by Vissers et al. (2015; Fig. 5), who find clear responses in Mg II k, C II, and Si IV slit-jaw images of the Interface Region Imaging Spectrograph (IRIS) to PMJs observed in Ca II lines. Emission of C II and Si IV towards the top of PMJs suggests heating to transition region temperatures.

Reardon et al. (2013) study transients in a sunspot penumbra from spectral imaging data in the Ca II 8542 line. Aided by cotemporal Hinode Ca II H imaging, they identify several PMJs in their dataset. The Ca II 8542 line profiles show enhanced emission in the wings out to $\pm 0.5 \AA$ with peaks at about $\pm 0.3 \AA$ and a line core that shows little difference compared to the surroundings. They point out the similarity with Ca II 8542 spectral profiles of Ellerman bombs, for example as shown in Vissers et al. (2013).

In this study, we expand on the observational characterization of PMJs by analyzing a high spatial-resolution time series of both narrow-band Ca II H filtergrams and spectral imagery in Ca II 8542. We employ an automated detection scheme to built a large statistical sample of PMJs. The detection scheme neatly takes advantage of the many sampling positions in the Ca II 8542 line, utilizing the full line profile. The dimensionality of the observations in wavelength positions is first reduced by employing principal component analysis (PCA), and is then used in detections performed employing the k-nearest neighbour (k-NN) algorithm, followed by object tracking and statistical analysis. 

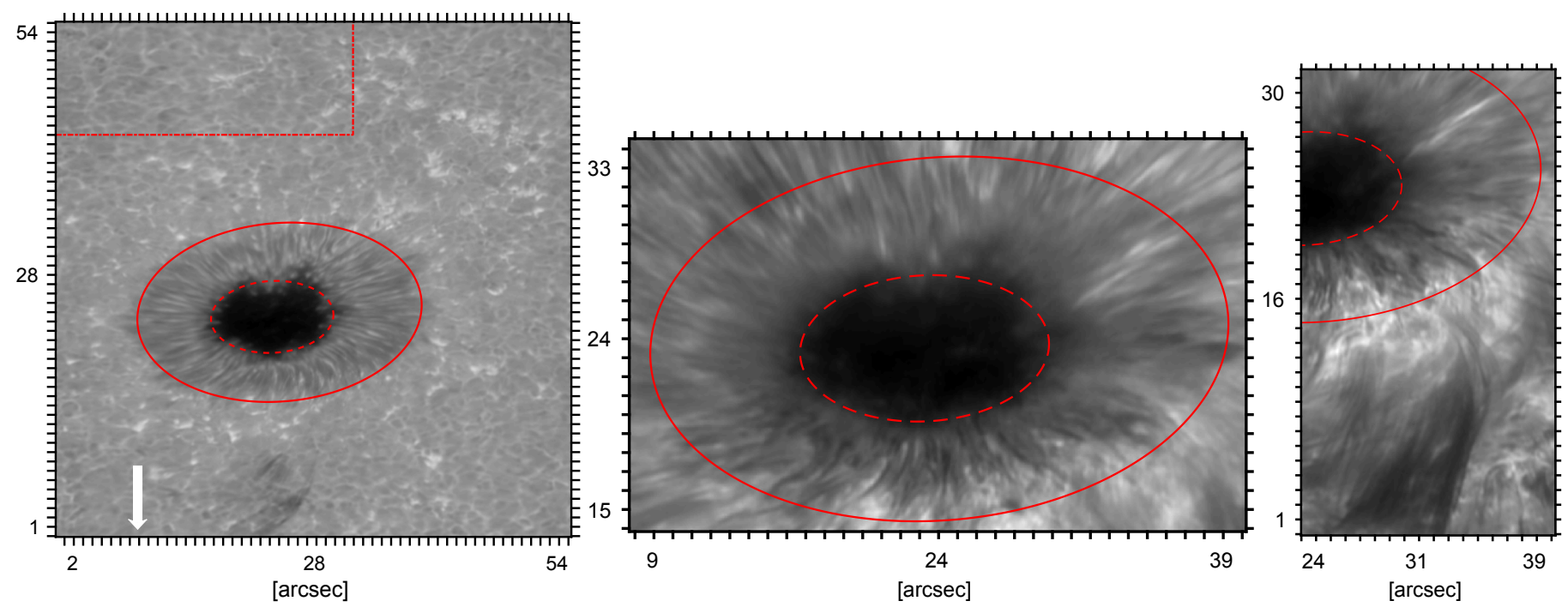

Fig. 1. Sunspot imaged by the SST on 28 June 2010 in the Ca II $8542 \AA$ line at offsets $-1032 \mathrm{~m} \AA$ in the full field of view (left) and $-275 \mathrm{~m} \AA$ (middle and right) in the CaII $8542 \AA$ line, zoomed in on the middle panel and a subfield that contains a strong inverse Evershed flow, which is the dark feature extending from the bottom of the frame to the edge of the penumbra (right). The borders of the umbra (dashed-red) and penumbra (solid-red) are indicated. Also indicated (left) is the area over which the reference quiet-Sun Ca II $8542 \AA$ average line profile was computed (dashed-dotted-red). The arrow (solid-white) indicates the direction towards disk-centre. Images on the left and at the middle are at time 23 min $27 \mathrm{~s}$, image on the right is at $37 \mathrm{~min} 46 \mathrm{~s}$.

\section{Observations}

Active region AR11084 was observed on 28 June 2010 with the Swedish 1-m Solar Telescope (SST, Scharmer et al. 2003a) on La Palma. The field of view was centred on the nearcircular sunspot with fully developed penumbra at heliocentric coordinates $(X, Y)=(710,-339)(\mu=\cos \theta=0.55$, with $\theta$ the observing angle). The seeing was excellent for the full 41 min duration of the time series, which started at 09:18:29 UT, and the image quality further benefited from the adaptive optics system (Scharmer et al. 2003b) and image reconstruction with the Multi-Object Multi-Frame Blind Deconvolution method (MOMFBD, van Noort et al. 2005). We analyzed data from instruments on both branches of the optical beam: from the CRisp Imaging SpectroPolarimeter (CRISP, Scharmer et al. 2008) on the long-wavelength branch "red beam", and filtergram imaging in Ca II H on the short-wavelength branch "blue beam".

With CRISP, we sampled the Ca II $8542 \AA$ line at thirty-seven line positions, with equidistant $55 \mathrm{~m} \AA$ steps out to $\pm 880 \mathrm{~m} \AA$, and additional sampling at \pm 948 and $\pm 1034 \mathrm{~mA}$. CRISP has a full width at half maximum (FWHM) of $110 \mathrm{~m} \AA$ at $8542 \AA$, therefore the Ca II line is critically sampled throughout the central part of the spectral line profile. We acquired eight exposures per spectral sampling that were used for MOMFBD image reconstruction. In addition, single-wavelength spectro-polarimetric samplings of the Fe I $6302 \AA$ line were acquired. Unfortunately, due to erroneous calibration settings, the precise wavelength for this sampling was unknown, which resulted in noisy and effectively useless maps of the four Stokes parameters. The acquisition time for Ca II $8542 \AA$ was $8.1 \mathrm{~s}$ and the temporal cadence of the time series was $12.4 \mathrm{~s}$. After MOMFBD restoration of the individual spectral-line scans, the data was put together as a time series after correction for the CRISP prefilter (FWHM $9.3 \AA$ for $\mathrm{Ca} I I)$, compensation of the diurnal field rotation, rigid alignment, and destretching. We used early versions of the different procedures that were later put together as the reduction pipeline for CRISP data (de la Cruz Rodríguez et al. 2015) including the
post-MOMFBD correction for remaining small-scale seeing deformations that are due to the non-simultaneity of the sequentially recorded narrowband CRISP images (Henriques 2012). The effective field of view of the time series is $55^{\prime \prime} \times 55^{\prime \prime}$, with a pixelscale of $0{ }^{\prime} .059$ pixel $^{-1}$.

In the blue beam, synchronized filtergrams were recorded at a rate of 10.8 frames s$^{-1}$ in the $\mathrm{Ca}$ II $\mathrm{H}$ line core, with a filter FWHM of $1.1 \AA$, and with a wider passband filter, FWHM of $10 \AA$, at $\lambda=3954 \AA$, between the Ca II H and $\mathrm{K}$ lines. These two imaging channels were MOMFBD-restored to produce a time series with an effective cadence of half the CRISP data, $6.2 \mathrm{~s}$. The alignment to the CRISP data was performed by crosscorrelation of the red and blue wideband channels, which both show the photosphere.

Figure 1 shows example frames from the observations, displaying the sunspot at offsets $-1032 \mathrm{~m} \AA$ and $-275 \mathrm{~m} \AA$ in the Ca II $8542 \AA$ line. Also displayed are the nominal borders of the umbra and penumbra and the area over which the average quiet-Sun Ca II $8542 \AA$ line profile used for comparison was computed. A cropped image of the observations also showcases a strong inverse Evershed flow.

\section{Methods}

Prior to the employment of the pipeline described below, the observations were investigated using the CRisp SPectral EXplorer (Vissers \& Rouppe van der Voort 2012, CRISPEX), which was used to interactively browse the MOMFBD-reduced observations. CRISPEX was also instrumental in the by-eye assembly of the reference set of PMJ and non-PMJ objects in the observations for later employment of the k-NN algorithm.

We restrict ourselves to a qualitative description and focus only on the basic concepts and overall structure. For an in-depth treatment, the reader is directed to Drews (2014), Sect. 5, in which the full methodology for the automated detection scheme and detection process is described, and a full explanation of the subsequent object tracking is provided. 
A. Drews and L. R. van der Voort: Microjets in the penumbra of a sunspot
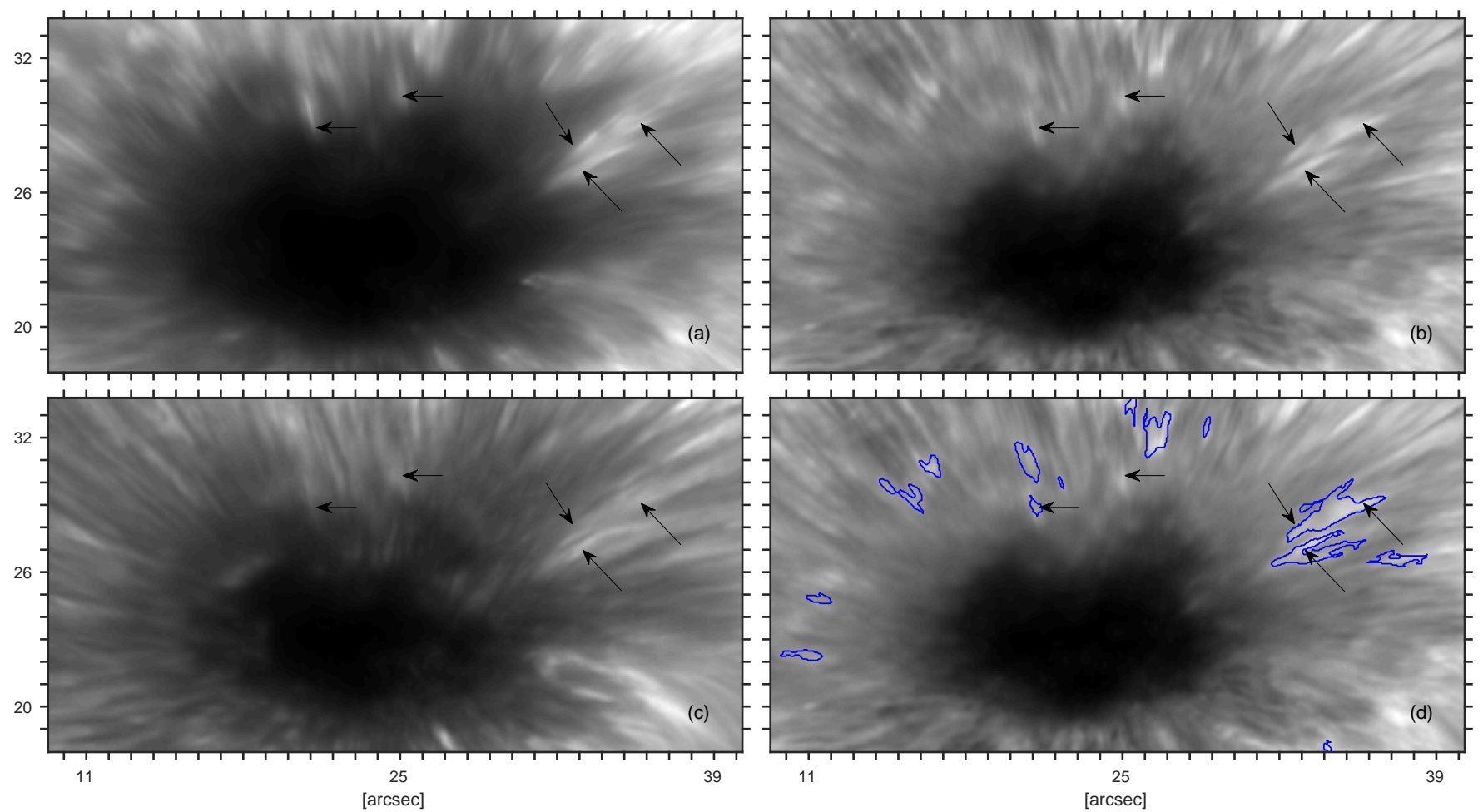

Fig. 2. Different wavelengths at time $25 \min 6 \mathrm{~s}$, with arrows at identical pixel positions indicating PMJ events a) Ca II H line core, FWHM $1.1 \AA$ A; b) Ca II 8542 at $-275 \mathrm{~m} \AA$ offset from line core, FWHM $110 \mathrm{~m} \AA$ in the Ca II 8542 line; c) Ca II 8542 line core; and d) Ca II 8542 at $-275 \mathrm{~m} \AA$ offset from line core with the detection borders of automatically detected PMJs in the FOV overplotted (solid-blue).

Covered in some more detail in the subsections below, the main working steps, starting from the post-MOMFBD SST observations, can be summarized as the following discrete steps:

1. preliminary identification of PMJs in CaII $8542 \AA$ using $\mathrm{Ca}$ II $\mathrm{H}$ lineobservations as reference;

2. principle component analysis: dimensionality reduction and data compression;

3. detection of PMJs using the k-nearest neighbour algorithm;

4. object tracking and statistical analysis.

\subsection{Preliminary identifications of penumbral microjets}

To justify the claimed observation of PMJs in the Ca II $8542 \AA$ line, a subset of observations was first compared to by-eye detections of PMJs in the co-observed $\mathrm{Ca}$ II $\mathrm{H}$ line, because the detection of PMJs and their appearance is firmly established in this line (Katsukawa et al. 2007; Jurčák \& Katsukawa 2008; Katsukawa \& Jurčák 2010; and Jurčák \& Katsukawa 2010). Earlier observations of PMJs in Ca II $8542 \AA$ were presented by Reardon et al. (2013), Drews (2014), as well as by Vissers et al. (2015).

A quick qualitative study was enough to show that many, if not most, Ca II H PMJ detections have spatially coinciding similar features in the Ca II $8542 \AA$ line, in particular slightly blue-ward of the nominal line centre wavelength. Similarly as in Ca II H, PMJs appear in selected spectral positions in the Ca II $8542 \AA$ line as short-lived, elongated brightenings in the sunspot penumbra.

This is illustrated in Fig. 2, which shows four example images in the observations at the same timeframe. One image is in the $\mathrm{Ca}$ II $\mathrm{H}$ line, one in the Ca II 8542 line core and two in the Ca II $8542 \AA$ line at an offset of $-275 \mathrm{~m} \AA$. Penumbral microjets proved to be most visible in by-eye detections in the Ca II $8542 \AA$ line in images at an offset of $-275 \mathrm{~m} \AA$. The arrows in the four panels all point to the same pixel positions, and it is clear that PMJ features are present in both diagnostics, namely in the Ca II H line and the Ca II $8542 \AA$ line scan. This is especially true when comparing features present in the $\mathrm{Ca}$ II $\mathrm{H}$ line which are clearly visible in the $-275 \mathrm{~m} \AA$ line offset Ca II $8542 \AA$ images as well. Detection borders of PMJs overplotted in panel (d) highlight that most by-eye selected examples in this frame were caught by the automated detection scheme that will be presented below in Sects. 3.2-3.4.3. Notably, two PMJs as selected by eye on the right of the field of view (FOV) are classified as one event by the automated scheme. Furthermore, one of the events is not detected by the detection scheme, as marked by the second arrow from the left in Fig. 2. Manual inspection using CRISPEX indicate that this event's spectral profile is not very distinct over a larger area.

From these examples, and other qualitative inspections of PMJs that visually coincided in space in the Ca II $\mathrm{H}$ line and in the Ca II $8542 \AA$ line observations, the assumption that these events are the same physical objects is validated. Further investigation of PMJs in the Ca II $8542 \AA$ line and the ascertainment of a distinct spectral line profile is therefore warranted.

\subsection{Principle component analysis}

The detection of PMJs in the presented automated approach is based on their distinct line profiles in the Ca II $8542 \AA$ line. Intensity differences throughout the field of view, which are due to both limb-darkening and local variations, proved to make detections using the k-NN algorithm difficult. Intensity variations affecting the whole line profile may not impact the shape of the given pixel's line profile, but may shift the overall intensity, 
including key sampling points in wavelength in which PMJs are visible, such that the similarity measures in the algorithm were less meaningful. For this reason, prior to PCA treatment, the observations were first normalized. The normalization consisted of normalizing each line profile in all pixels in all timeframes to its own sum. This preserved the line profile shapes, but removed any overall intensity variations effectively. The preservation of line profile shapes consequently carried over into the PCA-treated observations used in the k-NN detection scheme. Principle component analysis is both a method for data analysis, as well as being a useful tool for data compression. An introductory overview of PCA is given in Shlens (2014).

In PCA, the covariance between variables in a dataset is computed, constructing its covariance matrix. Subsequently, the associated eigen-vectors and -values of this matrix are found. The eigenvectors correspond to a new set of basis vectors along which the data can be projected, yielding a linearly independent dataset with no cross-correlation between variables. Normalizing the associated eigenvalues, these yield the relative contribution of the new basis vector variables to the total variance of the newly aligned dataset. In practice, this variance can be equated to the informational contribution from the given new eigenvector. Therefore PCA presents the opportunity for compression of a dataset, because discarding variables along eigenvectors with low informational value does not yield significant information loss overall, and additionally, this loss is quantifiable.

In the present context, the 37 wavelength sample points of the Ca II $8542 \AA$ line were treated as the variables in a 37-dimensional dataset. Hence, this was the dataset that was analyzed and compressed using PCA as outlined above. For the present pipeline, the computation of the covariance matrix of the Ca II $8542 \AA$ line observations was performed following Bennet et al. (2009), in which a numerically stable single-pass algorithm is presented. It provides the benefit of a lighter numerical workload wheras a naive, but generally stable, approach requires two passes over the dataset. In this kind of an approach, the mean of a dataset is first computed, followed by the computation of the needed powers of this mean in the second pass. The employed single-pass of Bennet et al. (2009) also avoids common numerical pitfalls in the computation of the covariances in terms of numerical instabilities when they are calculated in a single-pass approach. Using the found eigenvectors and corresponding eigenvalues, it was determined that the variables along seven eigenvectors describe the original observations to an accuracy, or informational content, of $97 \%$, which was deemed acceptable, whilst yielding a compression of the data to $19 \%$ of the original size. Thus, these seven eigenvectors were chosen as the principle components of the dataset, and the observations were aligned and compressed along them.

Different morphological features, all with distinct spectral profiles, such as long fibrils, umbral flashes and fibrils with strong flows, are clearly identifiable in maps of the different principle components. This lends intuitive credence to the PCA reduction method, in that the different "new spectral" variables still represent real features picked out from the original sampling positions. For examples, we refer to Drews (2014), Sect. 5.

\subsection{The k-nearest neighbour algorithm}

The k-nearest neighbour algorithm is conceptually simple, yet powerful. This is one of the reasons it is widely applied in signal-processing tasks such as facial and voice recognition as well as machine reading. For an introduction to the specifics of the algorithm, an overview and discussion for improvement of the algorithm is given in Guo et al. (2004). Furthermore, Yang \& Liu (1999) compare the algorithm to other classifiers.

Often termed the simplest of the machine learning algorithms, the k-NN algorithm is based on a comparative approach in which the data to be classified is related to a pre-classified reference set of the same type. In practice, this means that a reference set is assembled using expert knowledge or a manual classification scheme, and is then used to classify the rest of the data using a similarity measure. We employed a simple euclidean metric in the 7-dimensional PCA-reduced dataset. A reference set was assembled using by-eye detections, noting the temporal and spatial location in the observations. At present, a reference set corresponding to a total number of 958 positions in time and space was assembled. This reference set is further divided into $168 \mathrm{PMJ}$ positions, comprised of 55 separate events, and 790 background positions of large diversity. These specific numbers were found to yield robust results following a trial-anderror approach, during which we studied the number and variability of reference events needed until the results were satisfactory and consistent. Because the reference set is polled for each automatic identification, an unnecessarily large reference set is to be avoided.

The background positions correspond to datapoints that are clearly not PMJs. The k-NN algorithm uses both object and non-object entries in the reference set, such that each vector in the PCA reduced data has its associated distance computed to all points in the reference set for identification. A number of k-nearest neighbours are then polled, and the point is classified by majority vote. The selection of the parameter $\mathrm{k}$ is performed using an accuracy test of cross-classification of the assembled reference set. The classifications resulted in binary maps for each timeframe, consisting of background and PMJ detections that were then further processed, as outlined in Sect. 3.4. Figure 3 shows the Ca II $8542 \AA$ line profiles of the individual PMJs and background, or non-PMJ positions, that were assembled for the $\mathrm{k}-\mathrm{NN}$ reference set. It bears remarking that these profiles are separated for the sake of clarity for the two plots, but form one reference set in practice, with profiles marked as PMJ or non-PMJ in the set when polled. The selected PMJ profiles have a wide span in terms of intensity throughout the line, but are well-defined by their shape. This is also illustrated by their included average, which presents itself as a very well-defined PMJ-like profile, as will be made clear in Sect. 4. The non-PMJ profiles span a wide variety of profiles, sampling positions in the quiet Sun, penumbra, umbra, and features such as the strong inverse Evershed flow present in the observations.

\subsection{Object tracking, statistical analysis and extraction of the Call $8542 \AA$ line profile}

The post-processing of the binary maps of raw PMJ detections output by the k-NN algorithm was performed in several steps, which are described below. First, the binary maps were cleaned of noise and objects tracked through time, as described in Sect. 3.4.1. Following this, base statistics were extracted from the basis of these tracked objects, as described in Sect. 3.4.2. Lastly, line profiles in the Ca II $8542 \AA$ line were found, and this process is detailed in Sect. 3.4.3. 

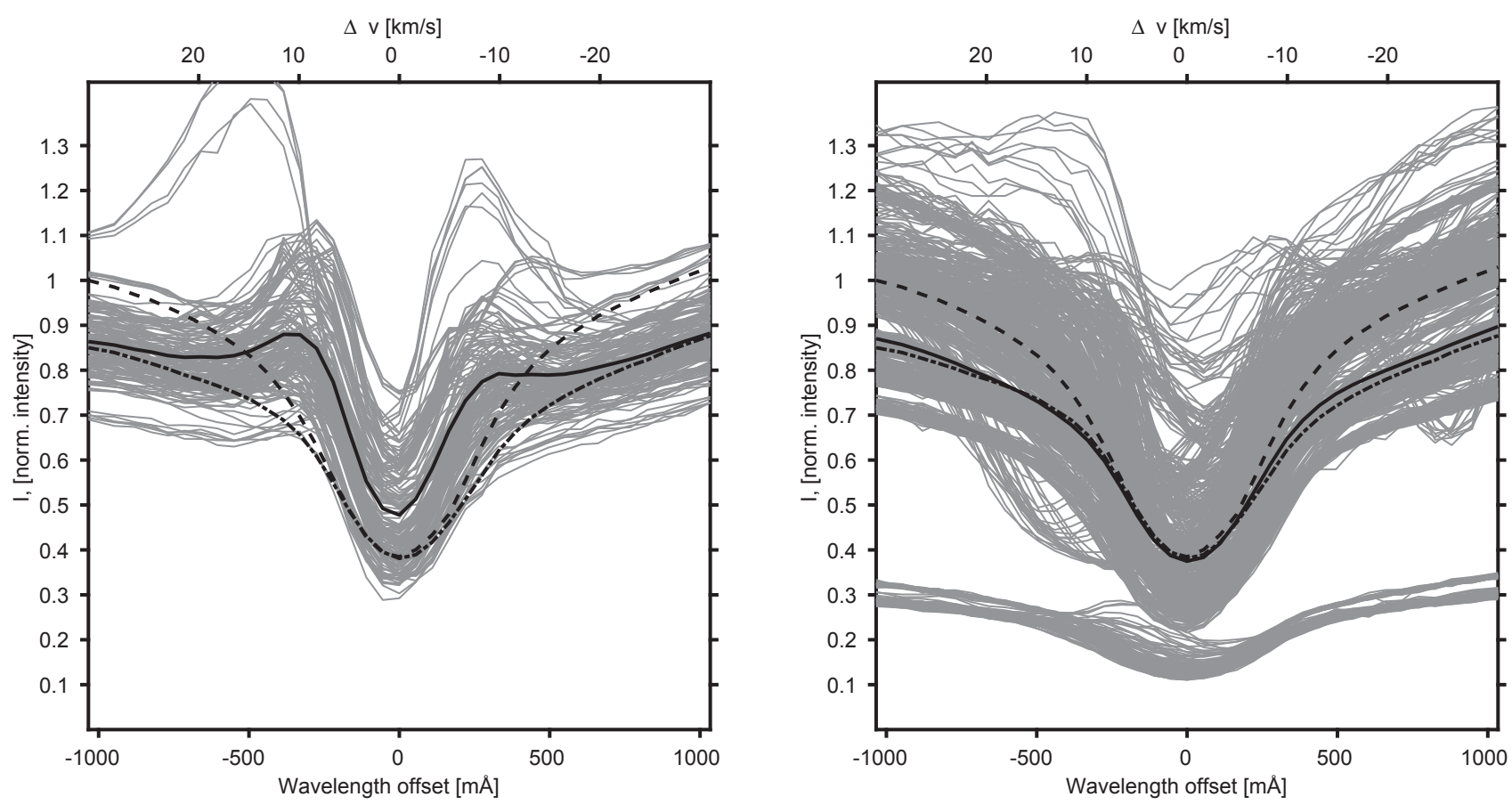

Fig. 3. Line profiles in the Ca II $8542 \AA$ line in the k-NN reference set used in the automated detections of PMJs. (Left) All 168 Ca II $8542 \AA$ line profiles of by-eye selected positions of PMJs used in the k-NN detection scheme (solid-grey) plotted together with their average (solid-black). (Right) All 790 line profiles of non-PMJ reference positions plotted together with their average (solid-black). For reference in both panels, the averages of the Ca II $8542 \AA$ line profile over the whole time series are given for the upper left corner of the full-FOV (dashed-grey) and the penumbra (dashed-dotted-grey) (see Fig. 1 for outlines of both areas).

\subsubsection{Noise removal and object tracking}

To remove spurious signals, the binary detections output from the k-NN algorithm were first run through an 8-pixel connectivity mask, removing lone falsely identified pixels. Furthermore, a lower "hard" area limit of 20 pixels was implemented on object sizes. Additionally, a "soft" area limit of 50 pixels was also implemented. This means that any areas in the original bitmaps below a size of 20 pixels total were not considered at all. The soft limit meant that if no area of a given tracked object reached a size of 50 pixels during tracking, the tracked areas were discarded. This means that tracked objects could have sizes of below 50 pixels, but only as long as the given object reached a size as large, or larger, than 50 pixels in at least one timeframe. This was meant to ensure that it was possible to catch the smaller beginnings of objects, but to avoid tracking spurious signals that never developed, and thus avoid noise from being tracked for long periods.

These area limits would correspond to cubic areas with sides 4.47 pixels $=194 \mathrm{~km}$ and 7.07 pixels $=307 \mathrm{~km}$ for the hard and soft limit respectively, although because a fitting of ellipseshapes is later performed (see below) the spatial limits along one axis may be smaller. The limits given may seem prohibitively large, but are chosen after trial and error and yield reasonable results. The final limits were chosen because lower values allowed small-scale noise in the bitmaps to be tracked and erroneously labeled as PMJs. This noise was mostly caused by a prominent inverse Evershed flow (see Fig. 1) in the lower right of the observations.

The object tracking was performed using relatively simple measures of spatial distances in the observations to relate discrete detections across timeframes. We used a maximum distance between centre-of-mass pixels of 15 pixels $=652 \mathrm{~km}$ between timeframes. This is equivalent to a projected speed of approximately $52 \mathrm{~km}^{-1}$, although it must be kept in mind that due to the morphing of detection areas and thus shifts of centreof-mass pixel positions between frames, this threshold can not be seen as a direct threshold on real movement of detected PMJs. Detections were also restricted to an outline of the photospheric penumbra, as seen in the wings of the Ca II $8542 \AA$ line, see Fig. 1. Only objects with centre-of-mass pixels inside this outline were included in the subsequent analysis. Furthermore, spurious detections inside an outline of the umbra were also discarded, see Fig. 1. These events were most likely related to umbral flashes, as was evident when investigating using CRISPEX.

\subsubsection{Basic statistics extraction}

A statistical analysis on the resultant final PMJ detections was then carried out. For estimations of detected object sizes, a fitting of ellipses on the detected objects was carried out. The reasoning behind this was the assumption that jet-like objects such as PMJs should in principle exhibit a somewhat elongated shape. The tracking was thus restricted to PMJs corresponding to bestfit-ellipses with an eccentricity of 0.9 , corresponding of an approximate ratio of semi-major to semi-minor axis in the ellipses of 2.3:1. The associated major and minor axes of these fitted ellipses for each single object were employed as estimates for the lengths and widths of the given detections respectively (see Fig. 4 for examples of these fitted ellipses). This allowed for the collection of size-statistics. Angular positions around the centre of the sunspot for detected objects were also computed.

\subsubsection{Computing the line profile}

The "master" average line profile for PMJs in the Ca II $8542 \AA$ line was computed using the line profiles of the centre-of-masspixels and their 8 neighbour-pixels for each individual detection. 
The average was thus carried out for only those PMJ detection areas through all timeframes that actually contained the centreof-mass-pixel, as computed from the detection area for each detection, and this pixel's 8 pixel-neighbours. This selection was performed to ensure that only line profiles of pixel positions that did not fall outside of the actual PMJ detection areas, and additionally that only pixel positions that represented well-formed PMJs, were used. The former case of a centre-of-mass pixel falling outside its associated detection area could occur due to for example peanut-shaped PMJ areas. The final average line profile is thus based on a subset of the 4253 PMJ detections through all timeframes. This totalled 3953 PMJ detections, because not all PMJ areas contained their theoretically computed centre-ofmass pixel, or because the area did not contain all 8 immediate pixel neighbours of the centre-of-mass pixel.

For the computation of the average line profile a total of $3953 \times 9=35577$ pixel-position line profiles was therefore used. The entirety of pixel positions of all PMJ detection areas were not employed for simplicity, and as not to skew the line profiles towards larger PMJs, that would then have contributed more strongly towards the profile. However, a difference in line profiles between large and small PMJs has not been investigated.

Finally, the individual 9-pixel-average line profiles of each of the PMJs in the mentioned subset were also investigated with regards to distinct peaks in the blue and red wing together with their line-core minimums. The wavelength positions of the peaks and the line-core minimum were estimated by interpolating the individual profiles, and then using a sliding-window approach to find the local peaks and minimum in each given profile. The final average values for the peaks and minimum were then based on the individually found peaks and minima. Profiles for the average of different categories of profiles, meaning profiles with blue and/or red peaks present, profiles with both blue and red peaks present and profiles with just either of the peaks present, were also computed to compare to the main average and each other. The resulting average values for the positions of the minimum, the blue and the red peaks were calculated by averaging the found positions in the individual interpolated line profiles, and are thus not limited to the thirty-seven sampling points. They are therefore subject to greater uncertainty.

\section{Results}

The automatic detection of PMJs as outlined in the last section made it possible to collect a large and statistically significant set of PMJ detections with associated properties that could then be investigated. In the sections below the different derived properties and statistical values associated with these events are presented.

\subsection{Detection summary}

Table 1 summarizes the detections performed by the automatic detection pipeline, together with the most basic statistics derived from them. It is evident that the automatic approach of identifying PMJs using their spectral profile yields a large dataset from which to infer PMJ properties. With a number of PMJs equalling 453 tracked events, derived statistical values are significant. Each individual frame in the detections contains an average of approximately $21 \mathrm{PMJ}$, and this rate highlights the continuous occurrence of PMJs throughout the observations. Because each of these detected PMJs is tracked through time, but
Table 1. PMJ detection statistics.

\begin{tabular}{|c|c|}
\hline Total PMJ detections (over all frames) [count] & 4253 \\
\hline Tracked PMJ objects [count] & 453 \\
\hline Mean PMJ detections per frame $\left[\frac{\# \text { events }}{\text { frame }}\right]$ & 21 \\
\hline Mean lifetime,tracked PMJs (all) [s] & 117 \\
\hline Mean lifetime, tracked PMJs $(<8 \mathrm{~min})^{a}[\mathrm{~s}]$ & 90 \\
\hline Mean length ${ }^{b}[\mathrm{~km}]$ & 640 \\
\hline Mean width ${ }^{b}[\mathrm{~km}]$ & 210 \\
\hline PMJ minimum position ${ }^{c}\left[\mathrm{~km} \mathrm{~s}^{-1}\right]$ & 0.14 \\
\hline PMJ blue peak offset ${ }^{c}\left[\mathrm{~km} \mathrm{~s}^{-1}\right]$ & -10.4 \\
\hline PMJ red peak offset ${ }^{c}\left[\mathrm{~km} \mathrm{~s}^{-1}\right]$ & 10.2 \\
\hline
\end{tabular}

Notes. ${ }^{(a)} N_{8 \text { min. }}=437 .{ }^{(b)}$ Based on ellipse fit. ${ }^{(c)}$ Based on the average of the 9-pixel average interpolated line profiles.

with unique associated properties in each timeframe, the number of individual PMJs summed over all timeframes is greater than the 453 tracked events. This number is 4253 PMJs present throughout all individual frames and corresponds to the 453 distinct, tracked events (see Table 1). However, some further selection was performed before some statistics were computed, namely line profile averaging as well as intensity and line profile feature investigations, see Sect. 3.4.3.

Figure 4 depicts a sample frame from the observations at an offset of $-275 \mathrm{~m} \AA$ in the Ca II $8542 \AA$ line with PMJ detections shown. Visible in the figure are the overlain PMJ detection areas and the associated computed ellipses that were used to measure the lengths and widths of the PMJ detections.

As a further reasonability check, a check of the relative brightness in the detection areas of all PMJs was performed. The individual average brightness in both the Ca II $8542 \AA$ line at an offset of $-275 \mathrm{~m} \AA$ and in the Ca II $\mathrm{H}$ line core was computed for each PMJ detection area. These were normalized to the average brightnesses for each individual frame of the penumbral region (as outlined in Fig. 1) in each of the two passbands. PMJs are seen as bright features in by-eye detections in both wavelengths, and thus one would expect an average brightening in the detection areas for both. Furthermore, as a result of the detections being carried out ultimately utilizing the Ca II $8542 \AA$ line profile, an average brightening in the detection areas in the $\mathrm{Ca}$ II $\mathrm{H}$ line core would further strengthen the co-occurrence of PMJs in both passbands. We find that $80 \%$ of PMJs have an average relative brightening above unity in the $\mathrm{Ca}$ II $\mathrm{H}$ line core and that $79 \%$ of PMJs have an average relative brightening above unity in the Ca II $8542 \AA$ line at an offset of $-275 \mathrm{~m} \AA$. Furthermore, $71 \%$ of detections show a simultaneous brightening above unity in both passbands throughout the times series. These values strengthen the assumption that bright features are being detected in the brightness-independent detection scheme utilizing the Ca II $8542 \AA$ line profile shape. Because the brightness of the PMJs was estimated as an average of their entire detection area, their brightness as compared to the average of the entire penumbra for a given timeframe may in fact be a conservative comparison. A comparison between, for example, the brightest pixel and the penumbra average for any given PMJ detection would likely yield a higher relative brightness in both passbands. This is due to automatically detected PMJs tending to exhibit a larger area than if selected solely by-eye compared to the surrounding intensity. Comparing PMJ brightnesses to the local average 


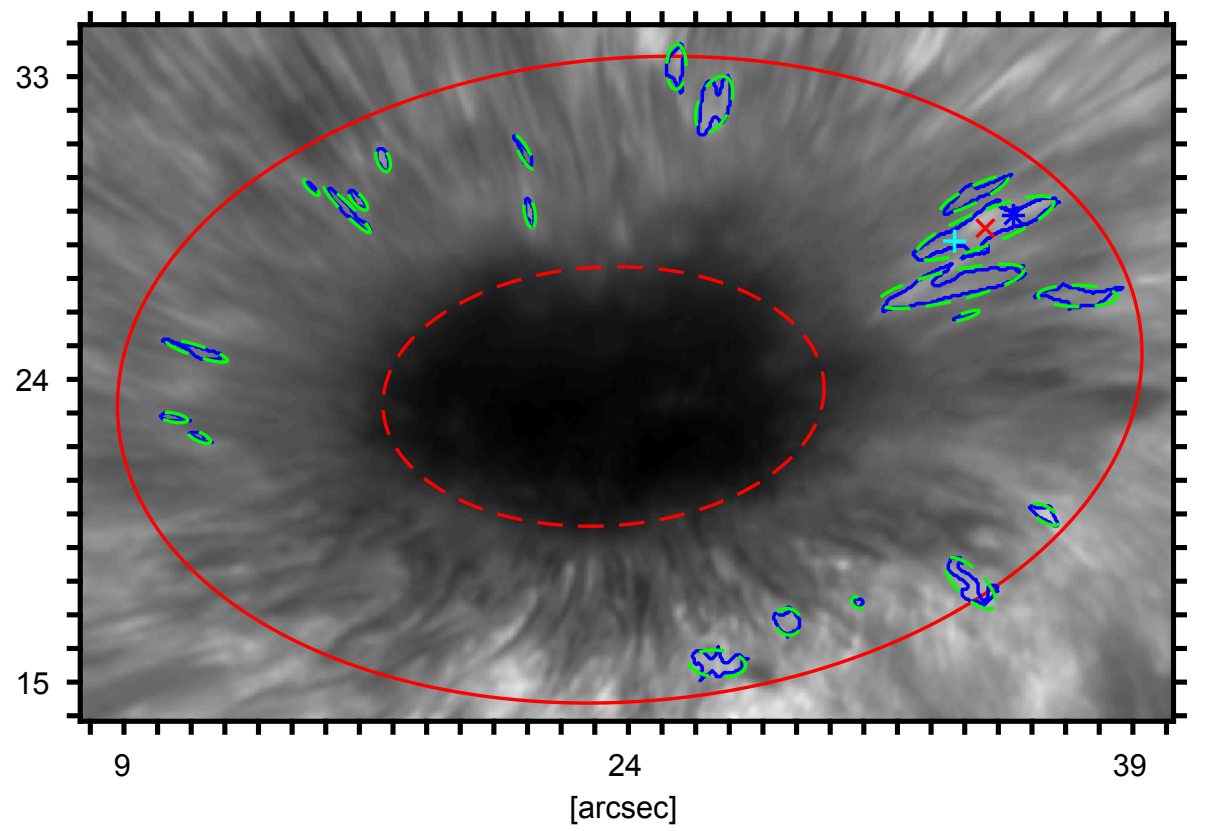

Fig. 4. Cropped image in the Ca II $8542 \AA$ line at an offset of $-275 \mathrm{~m} \AA$ with overlain PMJ detection areas (solid-blue) and corresponding computed best-fit ellipses (dashed-green) at time $23 \mathrm{~min} 27 \mathrm{~s}$. Also indicated for reference are three pixel positions inside one of the detection areas, one closer to the sunspot centre (turquoise cross), the PMJ centre-of-mass-pixel (red $\times$-mark), and one closer to the edge of the penumbra (blue star). Detailed line profiles for these pixels are shown in Fig. 13. brightness of the penumbra may very well have also resulted in a higher relative brightnesses.

\subsection{Basic penumbral microjet statistics and properties}

Having computed the length and width for all PMJ detections using the minor and major axis of the fitted ellipses (as described in Sect. 3.4.1), they could be presented as distributions. Figure 5 shows these histogram distributions for the lengths and widths of all detected PMJs throughout the observations.

The distributions for lengths and widths seem to be wellbehaved with trailing ends tending towards zero, as is expected. The lower ends of the distributions also seem to taper off in the last lower length and width bins. One pixel in the observations corresponds to $43 \mathrm{~km}$, thus both distributions' lowest length and width bins with entries include sizes larger than this. However, the detections were limited by a lower-limit area, that also effects the lower range. The average width and lengths must thus be interpreted with this in mind. The associated mean length of $640 \mathrm{~km}$ and mean width of $210 \mathrm{~km}$ (see also Table 1) are reasonable. They are also consistent in rough magnitude with values reported in Katsukawa et al. (2007), namely lengths of $1000-4000 \mathrm{~km}$ and widths of approximately $400 \mathrm{~km}$ or less for the PMJs in Hinode Ca II H observations. These values are also consistent with the mega-metre range as given in Reardon et al. (2013) when observing individual PMJs or penumbral transients in the Ca II $8542 \AA$ line. Both estimates found here are however still considerably smaller at almost half for both values. In Fig. 6 the distribution of durations of tracked PMJs is provided with a cut-off value of $8 \mathrm{~min}$, representing $96 \%$ of tracked detections, not showing outliers.

These outliers were excluded because, for the most part, they represented a small fraction of tracked PMJs that had extremely long lifetimes. The abnormally long lifetimes are most likely due to detections in areas in which PMJ detections were ubiquitous throughout the observations, and where PMJ events overlapped so closely in time and space that they were tracked continuously as one event. Also, spurious and long-lasting detections may also be caused by the strong inverse Evershed flow (see Fig. 1) present on the centre side of the observed sunspot.

\subsection{Ca II $8542 \AA$ penumbral microjet line profile}

The PMJ line profile in the CaII $8542 \AA$ line computed from the average of the $3953 \times 9=35577$ pixel-positions is given in Fig. 7, together with reference profiles for the quiet Sun and the penumbra. The PMJ profile is characterized by enhanced inner wings at about $\pm 385 \mathrm{m \AA}$ and a brighter line core that is at $116 \%$ of the quiet-Sun-line-core brightness. The enhancement of the inner blue wing is stronger than in the red wing. The far wings of the PMJ profile approach the level of the average penumbra. This average profile is less pronounced than individual profiles of PMJs selected by eye and by inspecting individual pixel-position profiles, as will be made more evident in Sect. 4.4.

The average PMJ profile that we find is however still consistent with other reported profiles as given in Reardon et al. (2013, their Fig. 4) and Vissers et al. (2015, their Fig. 5). Although it does show a less pronounced peak in the blue and a very weak enhancement in the red compared to most of these published profiles, the profile is still recognizable. It must be emphasized that the profile presented here is an average, computed from many individual profiles, wheras the profiles it has been compared to in previous work are profiles from individual pixel positions of byeye-selected PMJs. These individual profiles were most likely selected specifically due to their distinct features, meaning that the average profile presented consequently will present features that are less sharp. Individual profiles of PMJs in the presented observations, including both those selected by eye and those contained in PMJ detection areas, still exhibit such distinct features to a large degree, as will be exemplified in Sect. 4.4.

As mentioned in Sect. 3.4.3, when averaging the master line profile, each individual 9-pixel average profile corresponding to an individual detection was inspected for the presence of a telltale blue and/or red peak in the line. As a result, it was possible to create averages of the profiles according to the presence of peaks in the blue and/or red. Thus, averages for the profiles for the cases of both a blue and red peak being present, one or the other being present, or just one of the peaks being present could be computed. Figure 8 shows these average Ca II $8542 \AA$ line profiles for different subsets of PMJ detections. 

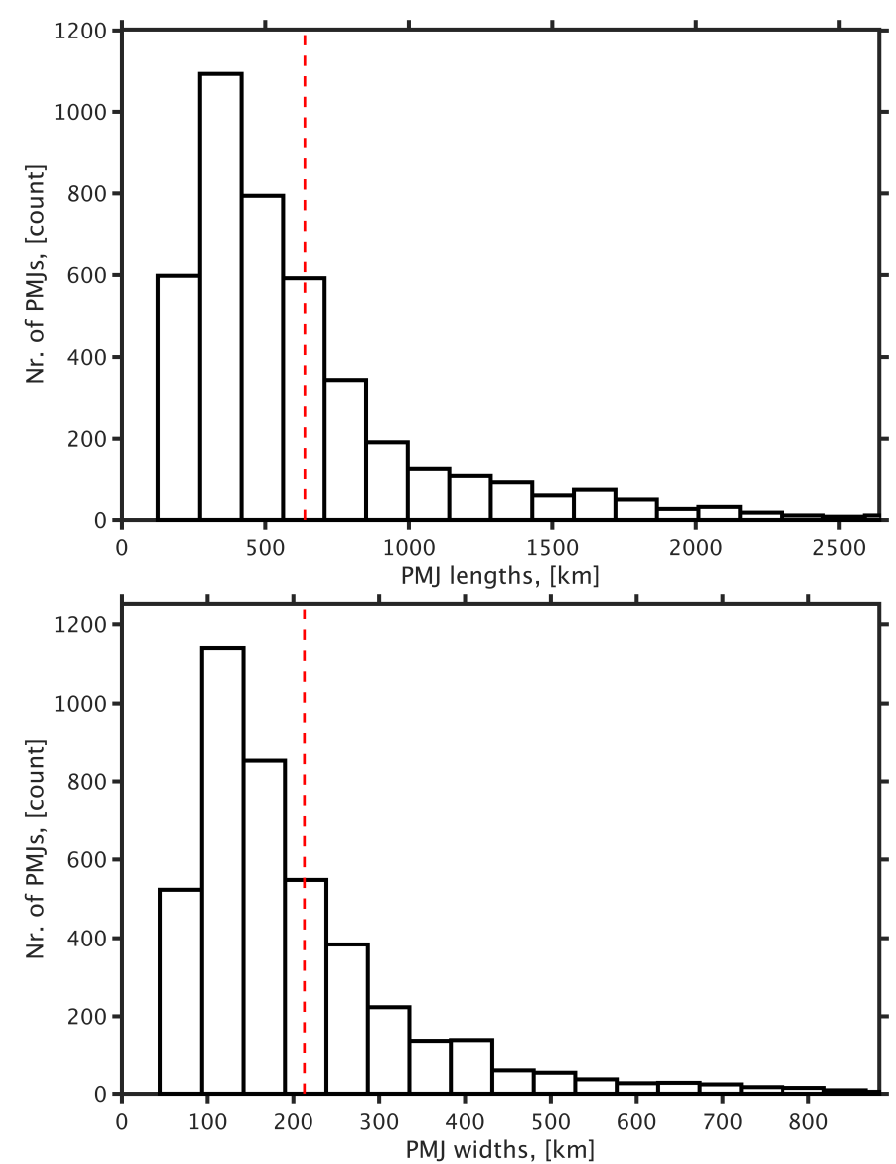

Fig. 5. Length and width distributions for PMJs throughout all timeframes. (Top) Length distribution with approximate bin size of $145 \mathrm{~km}$, with mean value (dashed) at $640 \mathrm{~km}$ indicated, the median value is $489 \mathrm{~km}$. (Bottom) Width distribution with approximate bin size of $48 \mathrm{~km}$, with mean value (dashed) at $210 \mathrm{~km}$ indicated, the median value is $165 \mathrm{~km}$. The total sample number of individual PMJs is $N=4253$.

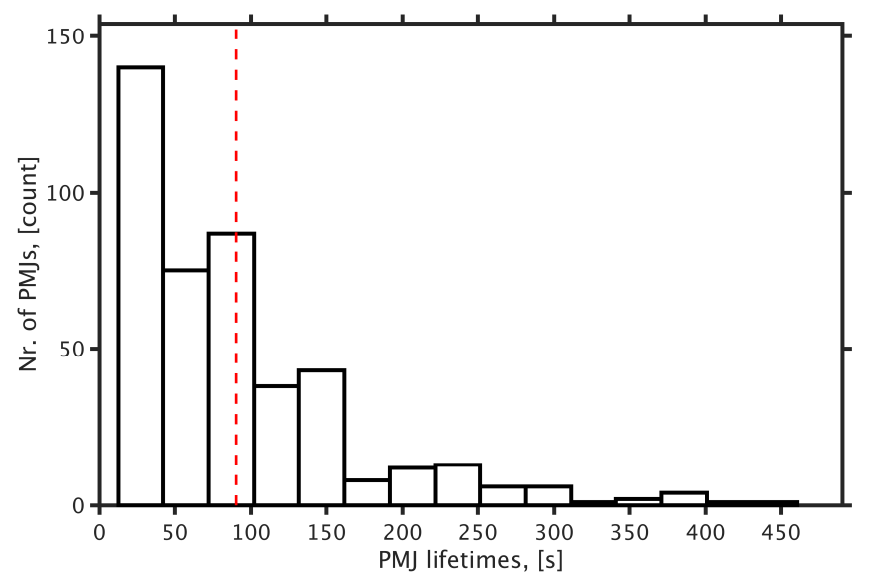

Fig. 6. Distribution of tracked PMJ lifetimes with an upper cutoff value of $480 \mathrm{~s}(8 \mathrm{~min})$ and bin size of $29.9 \mathrm{~s}$. $\mathrm{N}_{8 \text { min. distr. }}=437$ (96\% of total tracked PMJs). The mean value of $90 \mathrm{~s}$ is indicated (dashed-red), the median value is $75 \mathrm{~s}$.

For completeness, Table 2 summarizes these different groups of profiles. We especially note that only less than half, $N_{\text {profiles with peaks }}=1868$, of all 9-pixel average PMJ profiles used in the peak detection, $N_{\text {all }}=3953$, have clear automatically detectable peaks. Furthermore, a vast majority of these were in the blue, with a total number of blue peaks of $N_{\text {blue peaks }}=1725$

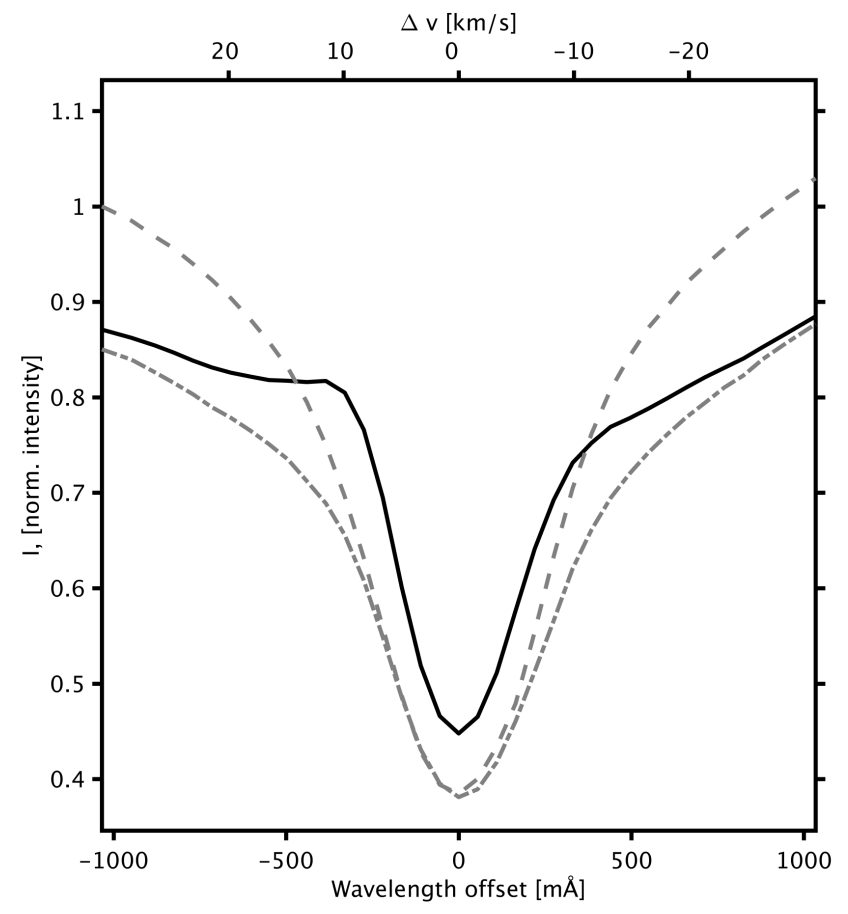

Fig. 7. Averaged Ca II $8542 \AA$ Aline profile of PMJ centre-of-mass pixels and their 8 neighbour-pixels (solid-black). For reference, the averages of the Ca II $8542 \AA$ line profile over the whole time series are given for the upper left corner of the full-FOV (dashed-grey) and the penumbra (dashed-dotted-grey). See Fig. 1 for outlines of both areas.

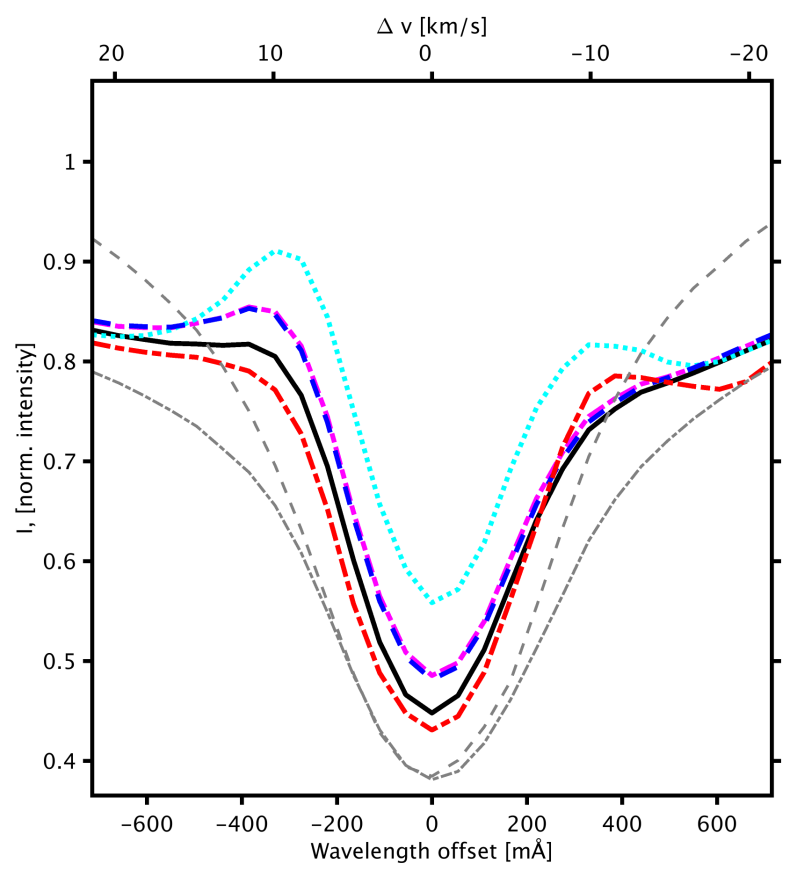

Fig. 8. Average Ca II $8542 \AA$ PMJ line profiles divided by the presence of clear inner-wing peaks: PMJs with only distinct blue peaks $\left(N_{\text {only blue }}=1637\right.$, dashed-blue $)$, PMJs with only distinct red peaks $\left(N_{\text {only red }}=29\right.$, dashed-dotted-red $)$, PMJs with both distinct blue and red peak simultaneously ( $N_{\text {blue and red }}=114$, dotted-cyan), and PMJs with distinct blue and possible red peaks $\left(N_{\text {blue with possible red }}=1725\right.$, dashed-dotted-magenta). We note that this profile nearly overlaps with the only-distinct-blue-peak profile. Included for reference are the profiles of Fig. 7: the full PMJ line profile average (solid-black), the average penumbra line profile (dashed-dotted-grey), and the average quiet-Sun line profile (dashed-grey). 
Table 2. Summary of PMJ peak-presence in individual profiles.

\begin{tabular}{ll}
\hline \hline $\begin{array}{l}\text { Nr. of (9-pixel average) profiles } \\
\text { used in peak detection }\end{array}$ & 3953 \\
Nr. of peaks detected & 1868 \\
\hline $\begin{array}{l}\text { Peak combinations } \\
\text { in profiles }\end{array}$ & Nr. of profiles \\
\hline Blue and/or red peak & 1780 \\
Both blue and red peak & 114 \\
& \\
Blue peak (with possible red) & 1725 \\
$\begin{array}{l}\text { Only blue peak } \\
\text { Red peak (with possible blue) }\end{array}$ & 1637 \\
Only red peak & 143 \\
\hline
\end{tabular}

and a total of red peaks of $N_{\text {red peaks }}=143$. These correspond to percentages of $92 \%$ and $8 \%$, of blue and red peaks respectively, of the total number of detected peaks. Furthermore, of all the investigated averaged 9-pixel profiles, $43 \%$ exhibit blue peaks whereas only $3.6 \%$ exhibit detectable red peaks.

From Fig. 8 it is evident that the average line profile of those profiles that have either both or one peak present (magentadashed-dotted in the figure) is near identical to the average line profile for those profiles that have blue peaks but no red peaks present (blue-dashed in the figure). This is concurrent with the fact that there is a much larger number of profiles with blue peaks compared to red peaks. The average line profile of profiles with only red peaks is correspondingly significantly different from both of these profiles and only shows a peak in the red. However we note the low number of twenty-nine profiles in this average. All three profiles are more distinct in appearance than the overall average line profile of all the 9-pixel line profiles (solid-black in the figure).

Finally, and most notably, the average line profile of those profiles with both blue and red peaks present simultaneously, which therefore has well-defined peaks in both the blue and red, more strongly resembles the profiles of by-eye selected PMJs in the present dataset. This will be made more evident in Sect. 4.4. For likely the same reason, this profile also more strongly resembles reported profiles found for by-eye detected PMJs in the Ca II $8542 \AA$ line given in Reardon et al. (2013, Fig. 4) and several of the distinct profiles presented in Vissers et al. (2015, Fig. 5). The average line profile of those profiles with both blue and red peaks present simultaneously is also of overall greater intensity in both the peaks and the line-core minimum compared to the overall PMJ average profile and the FOV average of the Ca II $8542 \AA$ line. In fact, the line core has a $145 \%$ intensity compared to the quiet-Sun-line-core average, which is a clearly greater enhancement than for the overall line profile average that, as given earlier, exhibited an intensity of only $116 \%$ compared to the quiet Sun. This makes it plausible that the higher overall intensity in these types of PMJs makes it easier to pick them out by eye and thus makes the presence of both peaks in these selections more likely, whereas the automatic detection presented may not be as susceptible to this bias after the initial selection of the k-NN reference set. An over-selection of PMJs with strong enhancements in the red wing of the Ca II $8542 \AA$ line may therefore be likely in by-eye detections.

It is worth noting that the average PMJ profile of the k-NN reference set shown in Fig. 3 exhibits a similar shape to the average profile of detected PMJs with both blue and red peaks

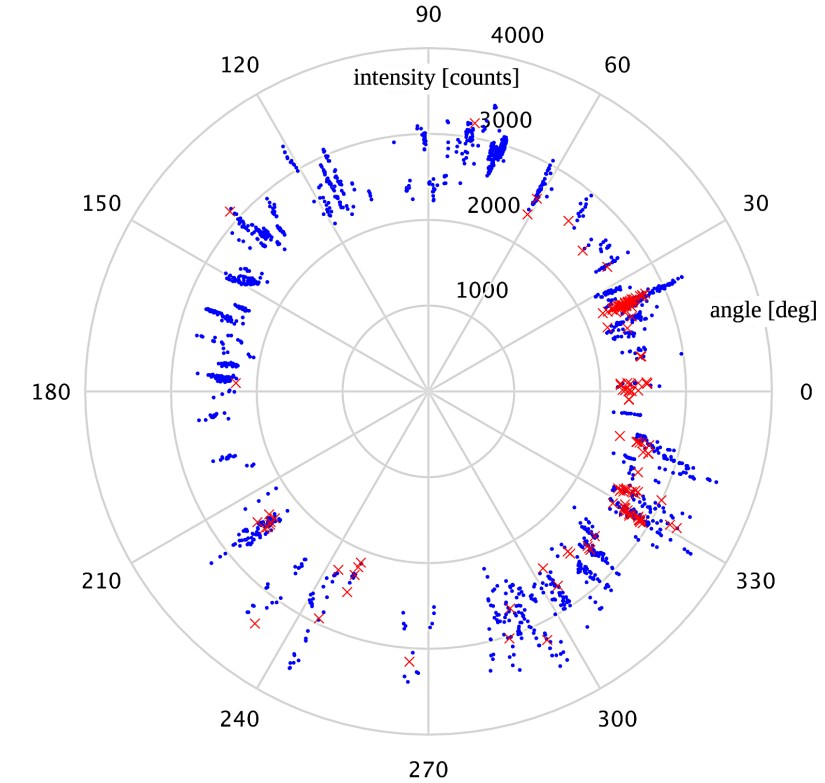

Fig. 9. Angular position inside the penumbra centred on the umbra, degrees along the polar axis vs. peak intensity in counts of blue and red peaks in PMJ profiles in the CaII $8542 \AA$ line. Plotted are the peakintensities in the respective wing for PMJs with automatically identifiable peaks in the red (crosses, $N=143$ ) and blue (dots, $N=1725$ ) wings of the line. The direction to disk centre is at approximately $270^{\circ}$.

present, and thus has more clear peaks than the final PMJ average profile of all PMJ detections. It also has an overall higher intensity than the total average, though not as high as the average with both blue and red peaks present. This similarity is most likely due by-eye selection favouring PMJs with strong enhancements in both the blue and the red as well as overall brighter PMJs, as previously raised.

We investigate the spatial distribution of the PMJ profiles with distinct blue and red peaks. It may be well plausible that the average PMJ orientation is related to the large scale magnetic field topology of the sunspot such that the inclined viewing angle may have an effect on the spatial distribution of the observed PMJ profiles. The results are presented in distribution graphs in polar coordinates, centred on the umbra and the limb direction at approximately $90^{\circ}$.

Figure 9 provides a scatter plot of the peak-intensities versus the angle around the centre of the sunspot of the automatically detected peaks in the red and blue of the Ca II $8542 \AA$ line for the detected PMJs. There is no clear discernible bias in the plot with regard to the intensity, both for the blue and red peaks. However, there is a readily apparent clustering of red peaks in the range of 315-30 degrees, with two noticeable groups within this range. The group above the zero-degree mark seems to neatly coincide with the principal PMJ hot-spot as described in Sect. 4.4 below (see Fig. 11). The presence of more readily detected red peaks in an area in which many PMJs are found throughout the time series is intuitive, because a higher count of PMJs should lead to a higher count of red peaks as well. On the other hand, the amount of red peaks seems over-represented in the area compared to other areas with significant numbers of PMJs, and may indicate that red peaks are favored in some areas.

In Fig. 10 we provide a scatter plot of the wavelengthpositions versus the angle around the centre of the sunspot of the automatically detected peaks in the red and blue of the Ca II $8542 \AA$ line for the PMJ detections. There is an apparent 


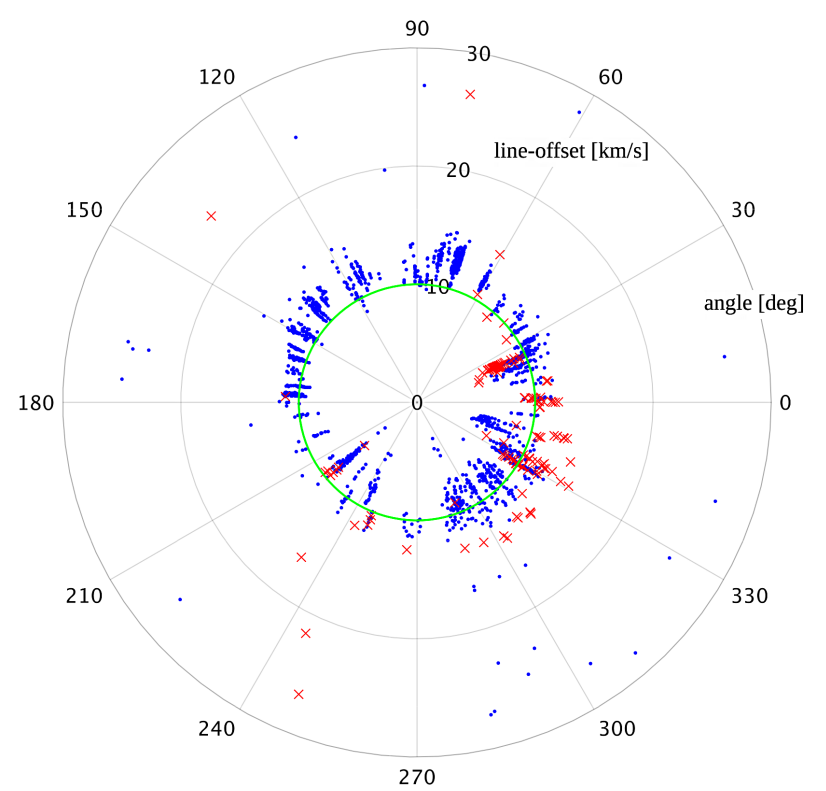

Fig. 10. Angular position inside the penumbra along the polar axis in degrees, vs. both absolute blue- and redshift from Ca II $8542 \AA$ linecentre along the radial axis in $\mathrm{km} \mathrm{s}^{-1}$. Plotted are offsets for PMJs with automatically identifiable peaks in the red (red crosses) and blue (blue dots) wings of the line. Also marked is a reference at $10 \mathrm{~km} \mathrm{~s}^{-1}$ (solidgreen).

bias in the degree of redshift in some of the red peaks, whereas there is no clear bias in the blueshift of the blue peaks. We can again discern the general clustering of red peaks in the same range as for Fig. 9, but this time there is a clear difference in the two groups within this angular range. The group coinciding with the PMJ hot-spot (see again Sect. 4.4 and Fig. 11) at slightly below 30 degrees has a clearly lower redshift and is less spread out in values than the group of red peaks clustered around 330 degrees (see also the reference line at $10 \mathrm{~km} \mathrm{~s}^{-1}$ in the figure). The atypical redshift of the red peaks situated around the 330 degree mark are likely largely caused by a strong inverse Evershed flow that moves into the penumbra at this location, which may also account for the greater overall spread of redshifts in this region. This region of strong inverse Evershed flow is an extension of the large dark cloud in the bottom middle part of the right panel of Fig. 1. We observe these inverse Evershed "clouds" moving into this part of the penumbra throughout the full duration of the time series. A detailed inspection of the spectral profiles of this region with CRISPEX reveals that at times the profiles are largely affected by this inverse Evershed flow.

There also seems to be an overall higher blueshift in the detected blue peaks of the Ca II $8542 \AA$ line profiles on the limb side of the Penumbra (in the 30 to 180 degree range). This is counter intuitive to a line-of-sight enhancement of the blueshift, because this would be expected for the disk side instead. This will be mentioned further in Sect. 5 .

\subsection{Clustering and a near-continuous occurrence of penumbral microjets}

Figure 11 depicts a density map of all the individual PMJ detection pixels, summed over all timeframes. Penumbral microjets are detected somewhat evenly distributed over the azimuthal direction and mostly in the outer half of the penumbra.
From the density distribution it is evident that there are preferred sites for PMJ formation and that they are not evenly distributed throughout the sunspot's penumbra. We can see a clear clustering of PMJ detections in certain regions, and two distinct regions in the upper right corner in particular. These two regions are the sites of a large number of PMJs throughout the observations.

There is an apparent bias in the number of detections with regards to position in the penumbra, as it is readily seen that there are many more detections on the limb side of the sunspot. The right-upper corner of the penumbra mostly coincides with the far side of the observer's line-of-sight, which might possibly contribute to a larger number of clear detections. Reciprocally, the lower side of the sunspot may exhibit a lesser amount of detections due to foreshortening of the nominally elongated PMJs. There are however still distinct hot-spots of PMJ-activity on the same side of the sunspot, meaning that a clustering of PMJs could not be caused by foreshortening effects to a large degree.

Figure 12 highlights the behaviour of a "PMJ-hot-spot", a site of repeated PMJ activity, that was picked out by-eye using CRISPEX (see legend for details). The timeslices in particular, and also the intensity-time plots, highlight how the PMJ-events seem to present as continuous processes of waxing and waning in intensity in both the Ca II $8542 \AA$ and the CaII H line, as opposed to very well-defined one-off events with clear onsets and ends. The event highlighted in these figures is situated in a PMJ-hot-spot as readily seen in Fig. 11. We can observe the typical behaviour of PMJs in the present observations, namely that they are localized to specific regions and seemingly reoccur over time. This ongoing process of PMJ generation at preferred sites may possibly be due to favourable magnetic field structures at these particular sites, leading to repeated magnetic reconnection in quick succession.

Figure 13 highlights the Ca II $8542 \AA$ line profiles of three specific pixel positions, all contained within a single PMJ detection area, as shown in Fig. 4. Shown in Fig. 13 are the profiles for a pixel position on the umbra side of the detection area, the centre-of-mass pixel position of the area, and a pixel position on the outer side of the area. These line profile examples highlight that the automatic detection envelops a larger area than that which would perhaps be picked out as a singular PMJ in by-eye detections (see Fig. 4), but that the contained profiles still have the distinct PMJ shape. In particular, this event may be categorized as two distinct jets using by-eye detections at the shown wavelength offset, whereas the automated method based on the line profiles identifies the entire area as one PMJ. The three different profiles highlighted all exhibit the distinct PMJ peak in the blue. There is also a clear enhancement in the red, but this is most evident for the centre-of-mass position and the reference point closest to the quiet Sun. The reference profile for the umbra-side position has some enhancement in the red, but the enhancement is less clear. The two profiles closest to the quiet Sun are very much distinct PMJ profiles, whereas the umbra-side profile is generally more subdued, but still with enhancement in the blue and red peak positions. The profile also has as a clearly enhanced line core compared to the penumbra average line profile. These three profiles are generally very similar to by-eye selected profiles (as for example seen in Fig. 12). The profiles in Fig. 13 also exhibit an incremental increase in intensity for the blue and red peak wavelength offsets as one moves towards the quiet Sun. This trend is not present for the line core however, because the line-core intensities remain fairly stable. 
A. Drews and L. R. van der Voort: Microjets in the penumbra of a sunspot

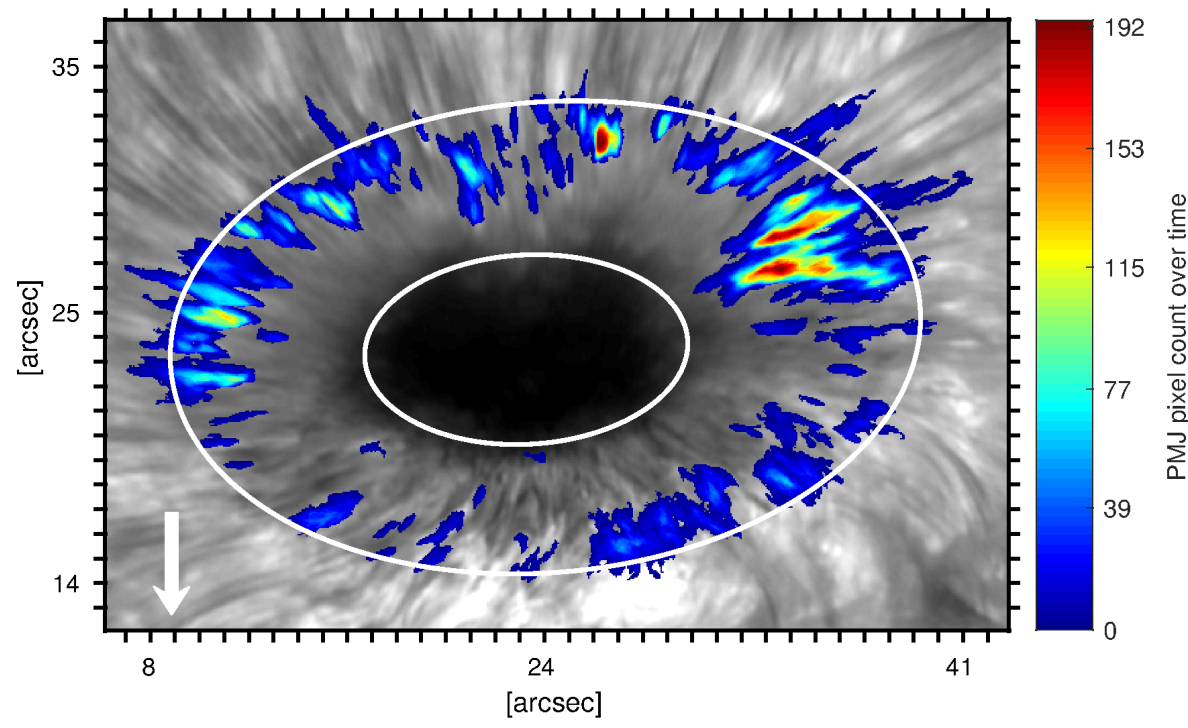

Fig. 11. PMJ densities, with all pixeldetections summed over all 202 timeframes, overlain onto a frame at the midpoint in time of the observations, at an offset of $-275 \mathrm{~m} \AA$ in the Ca II $8542 \AA$ line. The arrow indicates the direction towards disk-centre.
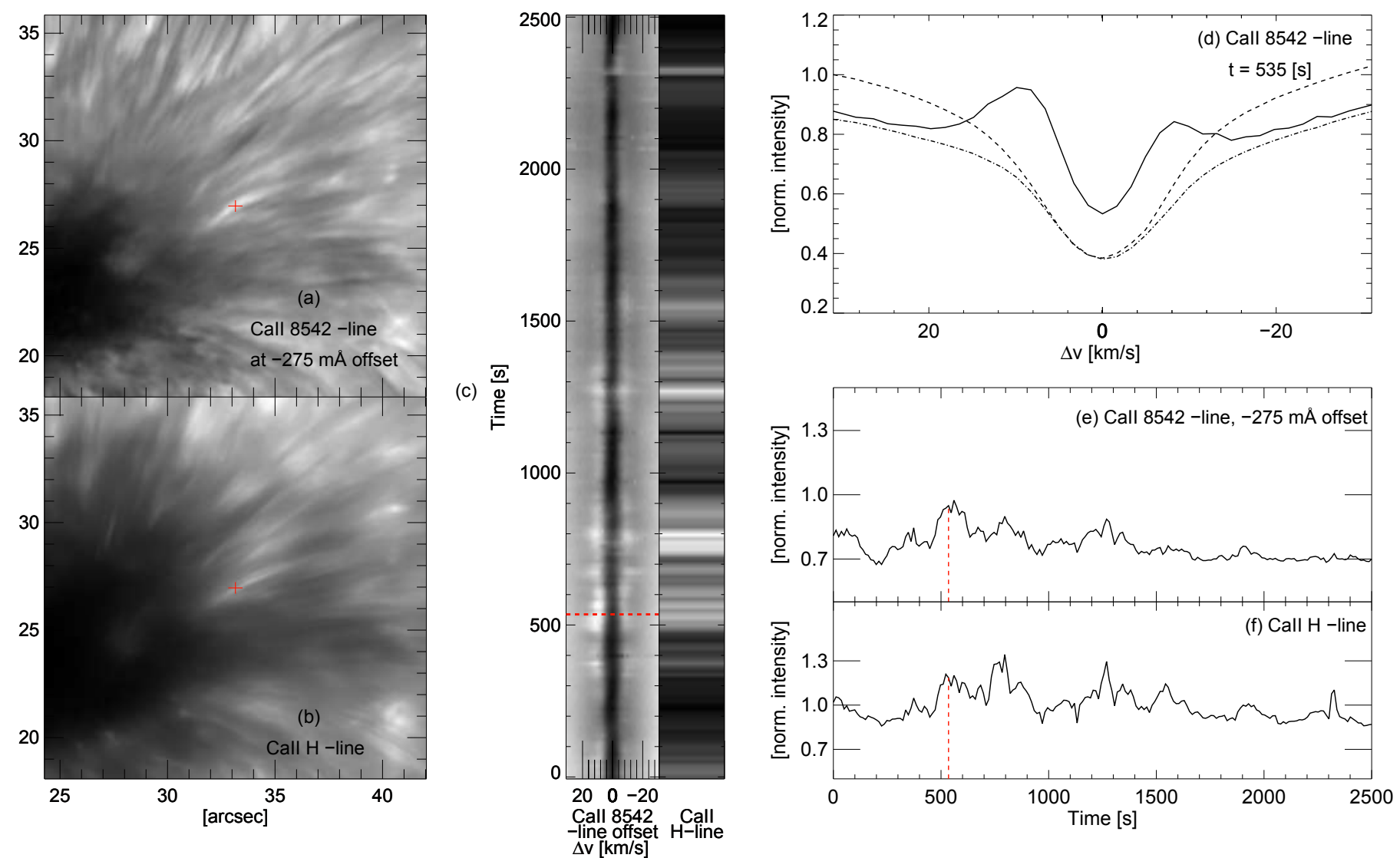

Fig. 12. PMJ event studied in detail at time $=535 \mathrm{~s}$. a) PMJ marked by a cross in a subfield of the observations in the Ca II $8542 \AA$ line at an offset of $-275 \mathrm{~m} \AA$. b) PMJ marked by a cross in a subfield of the observations in the Ca II $\mathrm{H}$ line. c) Timeslices for the full duration of the observations in the sampled Ca II 8542 A line (left) with time of the event indicated (dashed), and in the monochromatic Ca II H line core (right). d) Ca II $8542 \AA$ line profile of the PMJ event (solid) with the average of the line over the upper left section of the full FOV (dashed) and the average of the line over the penumbra, umbra excluded (dashed-dotted) (see Fig. 1 for these regions), with time of the event given. e) Intensity curve in the Ca II $8542 \AA$ line (solid) at an offset of $-275 \mathrm{~m} \AA$ for the full duration of the observation at the PMJ event location with PMJ event time (dashed). f) Intensity curve in the same position and duration in the Ca II H line core (solid) and event time (dashed).

\section{Discussion}

\subsection{Similarity to Ellerman bombs}

The typical PMJ Ca II 8542 spectral profile with enhanced inner wings resembles the characteristic spectral profile of Ellerman bombs, a similarity that was already pointed out by
Reardon et al. (2013) and Vissers et al. (2015). Ellerman bombs (Ellerman 1917) are the tell-tale signature of magnetic reconnection in the low atmosphere, usually associated with emergence of strong magnetic flux in active regions (see Rutten et al. 2013, for a recent review). Similarly to Ellerman bombs, magnetic reconnection is the driving mechanism for PMJs that is favored in the 


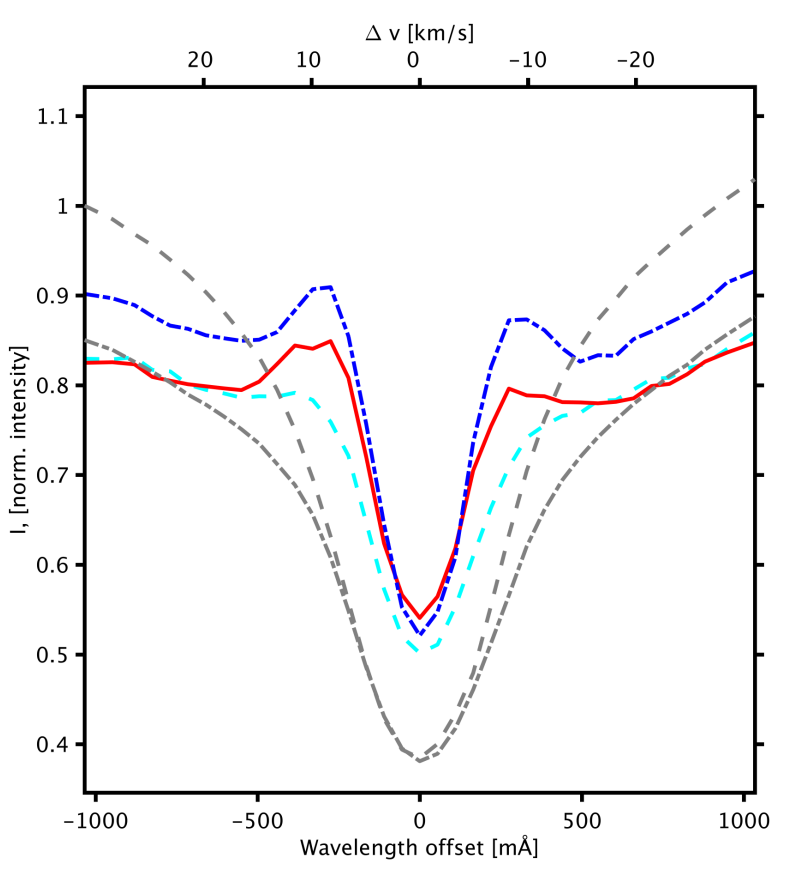

Fig. 13. Ca II $8542 \AA$ line profiles normalized to the average quiet-Sun line profile at different positions within a PMJ event detection area. The corresponding positions and the detection border of the event are marked in Fig. 4. Three specific PMJ line profiles are shown, one situated closest to the umbra (dashed-turquoise, turquoise cross in Fig. 4), the center-of-mass pixel position (solid-red, red $\times$-mark in Fig. 4) and one situated furthest from the umbra (dotted-dashed-blue, blue star in Fig. 4). Reference averages of the Ca II $8542 \AA$ line profile over the whole time series are given for the upper left corner of the full-FOV (grey-dashed) and the penumbra (grey-dot-dashed) (see Fig. 1 for outlines of both areas).

literature (see e.g. Katsukawa et al. 2007; Ryutova et al. 2008; and Nakamura et al. 2012). Ellerman bombs are commonly observed in $\mathrm{H} \alpha$ in which they exhibit enhanced wings but an undisturbed line core which is an indicator that the reconnection occurs below the chromospheric canopy (see, e.g., Watanabe et al. 2011). Vissers et al. (2013) study Ellerman bombs in $\mathrm{H} \alpha$ and Ca II 8542 from a CRISP data set that was acquired just before the data we present here. The Ca II 8542 Ellerman bomb profile has enhanced wings as in $\mathrm{H} \alpha$ (see their Fig. 5), and a dark line core that arises from obscuration by overlying chromospheric fibrils.

It is worth emphasizing that the wing enhancement in PMJs is much more modest than in Ellerman bombs, for which the intensity level in the inner wing rises above the far-wing quietSun level and reaches far into the wings, beyond a $1 \AA$ offset from the line core. In PMJs the enhancement extends mostly to about $0.6 \AA$ and stays well below the far-wing quiet-Sun reference intensity. Furthermore, we note a $116 \%$ intensity of the Ca II 8542 average PMJ line core as compared to the quiet Sun and a $145 \%$ intensity for the line profile average with both blue and red peaks present. In the line-core images, PMJs are not obviously obscured by overarching fibrils as is the case for Ellerman bombs, and sometimes we can discern bright features that resemble the PMJ morphology at $-275 \mathrm{~m} \AA$ offset, see the bottom panel of Fig. 2 for an example. We regard the enhanced line core as consistent with the observation that PMJs are heated to transition region temperatures, as reported by Vissers et al. (2015) who found signs of progressive heating along the PMJ length from coordinated IRIS and SST observations.

\subsection{Spatial distributions}

Besides the enhancement of both inner wings in PMJ line profiles, we note that there is a preference for larger enhancement of the blue wing. For those profiles in which the enhancement is in the form of one or two clearly identifiable inner-wing peaks, which number close to half of the detected PMJs, the majority have a blue peak. This is also reflected in the average PMJ profile that displays a clear blue-over-red asymmetry. We investigated whether there is any trend in the spatial distribution of the properties of the inner-wing peaks. If one would, perhaps naively, interpret PMJs as near-vertical plasma upflows, one would expect this to have an imprint on the observed spectral profiles for a sunspot under this observing angle of $\theta=57^{\circ}$. This viewing angle effect is for example very clear for the photospheric Evershed flow in sunspots, for which Dopplermaps show a clear, highly inclined, outflow in the form of redshifts at the limb side and blueshifts at the centre side for those sunspots that are away from disc centre (see, e.g., Scharmer et al. 2011, for a recent example). We find, however, no clear systematic imprint on the spatial distribution of spectral parameters of PMJ profiles. The peak intensity of the inner-wing peaks show no trend in the spatial distribution over the sunspot (see Fig. 9). There may be a trend of higher blueshifts of the blue peaks at the limb-side penumbra (Fig. 10) where the blue peaks are all shifted more than $-10.5 \mathrm{~km} \mathrm{~s}^{-1}$. On the centre-side, the shifts are between -7 and $-14 \mathrm{~km} \mathrm{~s}^{-1}$. This trend goes against the simple interpretation of the shift of the blue peak as a pure Dopplershift from a upflow in the expanding chromospheric magnetic field of the sunspot. In this kind of a scenario the strongest blueshifts would be found in the centre-side penumbra. We conclude that detailed numerical modelling with a realistic treatment of the radiative transfer in the optically thick penumbral atmosphere is required in order to interpret the PMJ spectral profiles. A complicating factor is the inverse Evershed effect in the form of mostly redshifted clouds that at certain times and spatial locations result in strongly affected line profiles. Part of the profiles that were identified as "red-peak" PMJ profiles were clearly affected by inverse Evershed clouds that were unrelated to PMJs.

\subsection{Spatial dimensions}

From our large statistical sample of automated PMJ detections, we determine PMJ lengths that are on the short side as compared to the measurements from Hinode $\mathrm{Ca} \mathrm{H}$ observations (Katsukawa et al. 2007). To a large extent this can be attributed to our method's sensitivity to weaker events and the inclusion of the centre-side penumbra, in which the projected PMJ extensions suffer from foreshortening. The measurements from previous studies have an intrinsic bias towards longer PMJs from by-eye selection.

\subsection{Lifetimes}

We determine an average lifetime of $90 \mathrm{~s}$ which is longer as compared to the typical lifetime of less than 1 min reported earlier (Katsukawa et al. 2007). However, we note that we find a large number of short-duration events, concurrent with the median lifetime of $75 \mathrm{~s}$ for the 8-min-cutoff PMJ lifetime distribution. We have discarded long-duration detections of $>8 \mathrm{~min}$. These were in part resulting from clustering of individual PMJs occurring in close vicinity and rapid succession, and in part due the earlier-described strong inverse Evershed flow on the disk side of the sunspot, that distorts the Ca II $8542 \AA$ line profile, and 
causes false identifications. Thus, we cannot exclude the possibility that intermediate duration detections, 3-8 min, are also affected by neighbouring PMJ activity. However, we decided to be conservative in manually sifting though the detection statistics. Furthermore, we again note our method's sensitivity to weaker events, and that this allows us to track events for longer durations as compared to manual and by-eye selection methods.

\section{Conclusions and summary}

We studied PMJs using an automated, simple machine-learning detection scheme consisting of an initial principle component analysis for the compression of data, a subsequent application of the k-Nearest Neighbour algorithm, and finally simple object-tracking over the time series. This scheme was applied to high-spatial-resolution observations of well-sampled Ca II 8542 profiles. We verify that the automated detections of PMJs in Ca II 8542 match well with PMJs in co-temporal Ca II H linecore filtergrams, which is the diagnostic used for PMJs in earlier studies. The Ca II 8542 PMJ line profile is characterized by enhanced inner wings, often in the form of clear peaks, preferably with a distinct asymmetry towards stronger blue-wing enhancement. The line core is enhanced as compared to the quiet-Sun reference spectrum. We detect a total of 4253 PMJs at a detection rate of 21 events per timestep over a duration of $41 \mathrm{~min}$, corresponding to 453 PMJs tracked in time. Ellipse fitting to the PMJ detection areas yields average PMJ dimensions of $640 \mathrm{~km}$ length and $210 \mathrm{~km}$ width. We measure an average lifetime of $90 \mathrm{~s}$, discarding the longest duration events of $>8 \mathrm{~min}$, which are clearly separate but overlapping events in rapid succession or the result of misidentifications. We detect PMJs in all parts of the penumbra, with many detections on both the limb side as well as on the disk-centre side of the penumbra. However, there was still an apparent bias in that there were more detections on the limb- or upper- side of the sunspot, perhaps in part caused by foreshortening effects. We note the existence of clear "hot-spots" with high occurrence rates of PMJs.

We finally remark that our results contribute to a solid observational characterization of PMJs that is needed to provide constraints for theoretical and numerical modelling. Further research will necessarily have to focus on numerical studies to elucidate the precise physical nature of PMJs. Quantification of the heat-energy transfer by PMJs into the higher sunspot atmosphere is one such area of interest for future investigations.
Acknowledgements. The Swedish 1-m Solar Telescope is operated on the island of La Palma by the Institute for Solar Physics of Stockholm University in the Spanish Observatorio del Roque de los Muchachos of the Instituto de Astrofísica de Canarias. Our research has been partially funded by the Norwegian Research Council and by the ERC under the European Union's Seventh Framework Programme (FP7/2007-2013)/ERC grant agreement No. 291058. We made much use of NASA's Astrophysics Data System Bibliographic Services.

\section{References}

Bennet, B., Grout, R., Pebay, P., Roe, D., \& Thompson, D. 2009, Proc. IEEE Int. Conf. Cluster Computing

Borrero, J. M., \& Ichimoto, K. 2011, Liv. Rev. Sol. Phys., 8, 4

de la Cruz Rodríguez, J., Löfdahl, M. G., Sütterlin, P., Hillberg, T., \& Rouppe van der Voort, L. 2015, A\&A, 573, A40

Drews, A. 2014, Master's thesis, Institute of Theoretical Astrophysics, University of Oslo, Norway, http: //urn . nb . no/URN : NBN : no-46622

Ellerman, F. 1917, ApJ, 46, 298

Guo, G., wang, H., Bell, D., et al. 2004, in Computational Linguistics and Intelligence Text Processing, 5th Int. Conf., CICLing 2004 (Seoul, Korea: Springer), 559

Henriques, V. M. J. 2012, A\&A, 548, A114

Jurčák, J., \& Katsukawa, Y. 2008, A\&A, 488, L33

Jurčák, J., \& Katsukawa, Y. 2010, A\&A, 524, A21

Katsukawa, Y., \& Jurčák, J. 2010, A\&A, 524, A20

Katsukawa, Y., Berger, T. E., Ichimoto, K., et al. 2007, Science, 318, 1594

Nakamura, N., Shibata, K., \& Isobe, H. 2012, ApJ, 761, 87

Reardon, K., Tritschler, A., \& Katsukawa, Y. 2013, ApJ, 779, 143

Rutten, R. J., Vissers, G. J. M., Rouppe van der Voort, L. H. M., Sütterlin, P., \& Vitas, N. 2013, J. Phys. Conf. Ser., 440, 012007

Ryutova, M., Berger, T., Frank, Z., \& Title, A. 2008, ApJ, 686, 1404

Scharmer, G. B., Bjelksjö, K., Korhonen, T. K., Lindberg, B., \& Petterson, B. 2003a, in SPIE Conf. Ser., 4853, eds. S. L. Keil, \& S. V. Avakyan, 341

Scharmer, G. B., Dettori, P. M., Lofdahl, M. G., \& Shand, M. 2003b, in SPIE Conf. Ser., 4853, eds. S. L. Keil, \& S. V. Avakyan, 370

Scharmer, G. B., Narayan, G., Hillberg, T., et al. 2008, ApJ, 689, L69

Scharmer, G. B., Henriques, V. M. J., Kiselman, D., \& de la Cruz Rodríguez, J. 2011, Science, 333, 316

Shlens, J. 2014, ArXiv e-prints [arXiv: 1404.1100]

van Noort, M., Rouppe van der Voort, L., \& Löfdahl, M. G. 2005, Sol. Phys., 228, 191

Vissers, G., \& Rouppe van der Voort, L. 2012, ApJ, 750, 22

Vissers, G. J. M., Rouppe van der Voort, L. H. M., \& Rutten, R. J. 2013, ApJ, 774, 32

Vissers, G. J. M., Rouppe van der Voort, L. H. M., \& Carlsson, M. 2015, ApJ, 811, L33

Watanabe, H., Vissers, G., Kitai, R., Rouppe van der Voort, L., \& Rutten, R. J. 2011, ApJ, 736, 71

Yang, Y., \& Liu, X. 1999, in A re-examination of text categorization (New York: ACM Press), 42 